\title{
Genome-Wide Analysis of the CCTGene Family in Chinese White Pear (Pyrus Bretschneideri Rehd.) and Characterization of PbPRR2 in Response to Varying Light Signals
}

\section{Zheng Liu}

Research Institute of Fruit and Tea, Hubei Academy of Agricultural Sciences

Jia-Li Liu

College of Life Sciences, Wuhan University

\section{Lin An}

Key Laboratory of Horticultural Plant Biology (Ministry of Education), Huazhong Agricultural University Tao Wu ( $\square$ wutaoga556@163.com )

Research Institute of Fruit and Tea, Hubei Academy of Agricultural Sciences

\section{Li Yang}

Research Institute of Fruit and Tea, Hubei Academy of Agricultural Sciences

\section{Yin-Sheng Cheng}

Research Institute of Fruit and Tea, Hubei Academy of Agricultural Sciences

\section{Xian-Shuang Nie}

Research Institute of Fruit and Tea, Hubei Academy of Agricultural Sciences

\section{Zhong-Qi Qin}

Research Institute of Fruit and Tea, Hubei Academy of Agricultural Sciences

\section{Research Article}

Keywords: Pear, CCT family, Phylogenetic analysis, Expression profile, Light environment, Transient overexpression

Posted Date: September 17th, 2021

DOl: https://doi.org/10.21203/rs.3.rs-885482/v1

License: (9) (1) This work is licensed under a Creative Commons Attribution 4.0 International License. Read Full License 
Version of Record: A version of this preprint was published at BMC Plant Biology on February 23rd, 2022. See the published version at https://doi.org/10.1186/s12870-022-03476-1. 


\section{Abstract}

\section{Background:}

Canopy architecture is critical in determining the light environment, and subsequently the photosynthetic productivity of fruit crops. Numerous CCT domain-containing genes are crucial for plant adaptive responses to diverse environmental cues. Due to the biological importance of $C C T$ genes, many researchers have focused on their functional characterization. However, little information was available about the CCT genes ( $P b C C T S$ ) of pear, an important fruit crop.

\section{Results:}

Genome-wide sequence analysis identified 42 putative PbCCTs in the genome of pear (Pyrus bretschneideri Rehd.). Phylogenetic analysis indicated these genes were divided into five subfamilies, namely, COL (14 members), PRR (8 members), ZIM (6 members), TCR1 (6 members) and ASML2 (8 members). Analysis of exon-intron structures and conserved domains provided support for the classification. Genome duplication analysis indicated that segmental duplication events played a crucial role in the expansion of the CCT family in pear, and that the CCT family evolved under the effect of purifying selection. Expression profiles exhibited diverse expression patterns of $P b C C T s$ in various tissues and in response to varying red and blue light. Additionally, transient overexpression of PbPRR2 in Nicotiana benthamiana leaves resulted in inhibition of photosynthetic performance, suggesting that PbPRR2 may be a negative regulator of photosynthesis.

\section{Conclusions:}

This study provides a comprehensive analysis of the $C C T$ gene family in pear and will facilitate further functional investigations of the PbCCTs to uncover their biological roles in light response.

\section{Background}

CCT [CONSTANS (CO), CONSTANS-LIKE (COL) and TIMING OF CAB1 (TOC1)] transcription factors, which encode proteins with a conserved motif (CCT domain) of $\sim 43$ amino acids residues towards their carboxy-terminus, constitute one of the plant-specific families $[1,2]$. The CCT domain has important functions in nuclear localization, protein-protein interactions, transcriptional regulation and nuclear protein transport [3-5]. CCT family genes can be divided into five categories based on the sequencing information of Arabidopsis: COL, PRR (Pseudo-Response Regulator), ZIM (Zinc-finger Protein Expressed in Inflorescence Meristem), TCR1 (Tunicamycin-induced COL-Related 1) and ASML2 (Activator of Spo ${ }^{\text {min }:: L U C 2) ~ s u b f a m i l i e s ~[6,7] . ~ T h e ~ C C T ~ f a m i l y ~ h a s ~ b e e n ~ c o m p r e h e n s i v e l y ~ a n a l y z e d ~ i n ~ s o m e ~ p l a n t s, ~}$ including Arabidopsis, rice, maize, brachypodium, sorghum, foxtail millet, barley, Aegilops tauschii and Medicago truncatula [7-11], but not yet in woody perennial fruit crops. 
It is well known that CCT family members play diverse and important roles in flowering, circadian rhythms, development and abiotic stress tolerance [12]. The COL subfamily has been extensively studied and can be further subdivided into three smaller groups, according to the degree of conservation and number of B-box domains [13-15]. In addition to the CCT domain, COL proteins contain one or two zincfinger B-box domains towards the amino terminus, thought to be involved in protein-protein interactions $[16,17]$. The first cloned CCT family gene in Arabidopsis is AtCO that required to promote photoperiodic flowering, at least partly by activating the expression of AtFT (FlowerT ime) and AtSOC1 (Suppressor of Overexpression of $\mathrm{CO1})$ genes [18-20]. Other $\mathrm{CO}$ homlogs $(\mathrm{COLS})$ also have been associated with abiotic stress tolerance, plant growth, development and metabolic processes, apart from playing a key role in the photoperiodic flowering induction [21-26]. PRR subfamily genes contain a Pseudo-receiver domain towards the amino-terminus, as well as a CCT domain at the carboxy-terminus [26]. They are key genetic components of the interconnected transcriptional-translational feedback loops, regulating circadian clock-output pathways [26-29]. In Arabidopsis, all PRR genes (AtPRR1, AtPRR3, AtPRR5, AtPRR7, and AtPRR9) which have redundant functions directly regulates the expression of genes implicated in abiotic responses, cell elongation, and photoperiodic flowering response [26-28,30]. In addition to the CCT domain, ZIM subfamily contains both a C2C2-GATA zinc-finger domain and a TIFY domain [31,32]. ZIM subfamily genes, including ZIM and ZIM-like (ZML) genes, are involved in hypocotyls and petiole elongation, cryptochrome 1-dependent response to excess light and wound-induced lignification [33-35]. TCR1 and ASML2 subfamilies represent two distinct classes of CCT family and both encode proteins possessing just a single CCT domain $[7,36]$. The function of TCR1 and ASML2 subfamily members are generally not well understood. AtTCR1 gene has been shown to be transcriptionally induced by Arabidopsis endoplasmic reticulum stress [7]. Overexpression of AtASML2 resulted in enhanced expression of a subset of sugar-inducible genes in Arabidopsis [36].

As sessile organisms, plants need depend on their ability to adapt to the complex changes of surrounding environment $[37,38]$. As a consequence, they have evolved power mechanisms that integrate environmental cues such as light signaling (light quality, light intensity and photoperiod), and their endogenous regulators to optimize their growth and productivity [30,39]. Pear is one of the most important economic fruit crops in the world. In the field, canopy architecture of pear is critical in determining the light environment, and thereby indirectly affects source-sink relationship [40]. To understand the potential mechanism of pear photosynthetic variability responses with heterogeneous light environment within canopies, we have conducted physiological and transcriptomic surveys to capture progressive stages of photosynthetic differentiation between distinct canopy structures [41]. We found that the two orthologs (LOC103943360 and LOC103951583) of AtPRR5 in pear were hub genes of the module positive correlated with pear photosynthetic rate, and may play key roles in photosynthetic performance under distinct canopy microclimate. There are increasing evidences that $C C T$ genes are associated with light responses and/or photosynthetic capacity. Ghd7, the homolog of CCT gene in rice, is a major locus responsible for natural variation of chlorophyll content at heading stage [42]. Another $C C T$ gene in rice, $C R C T\left(\mathrm{CO}_{2}\right.$ Responsive CCT Protein), controlled the capacity for starch synthesis, which can indirectly affect photosynthetic rate under elevated $\mathrm{CO}_{2}$ conditions [43,44]. In Arabidopsis, AtBBX4 is 
a CCT domain protein whose abundance positively modulated by phyB in red light, thereby promoting photomorphogenic development [45]. Considering the potential value of $C C T$ genes in light signal response and photosynthesis, we identified CCT genes (PbCCTs) in pear (Pyrus bretschneideri Rehd.) and analyzed the chromosomal locations, phylogenetic relationships and gene structure, as well as expression patterns of some members in various tissues and in response to different light signals. In addition, we performed functional analysis of one key $C C T$ member, PbPRR2, in transgenic Nicotiana benthamiana plants. This comprehensive study of the ССТ family may provide valuable information for the further research and utilization of the PbCCTs, helping to enhance our understanding of possible roles of $P b C C T s$ in the adaptation of pear to changing ambient light signaling.

\section{Methods}

\section{Genome-wide identification of CCT family genes}

The Hidden Markov Model profile of the CCT domain (PF06203) downloaded from the Pfam database (http://pfam.xfam.org/) was used for identification of the PbCCTs from the downloaded database of the Pear Genome Project (http://peargenome.njau.edu.cn/) using HMMER program (version 3.1b2) with a threshold of e-value $<10 \mathrm{e}^{-10}$ [46]. Using the same criterion, CCT family sequences from Arabidopsis thaliana and Oryza sativa were obtained from genome databases (ftp://ftp.ensemblgenomes.org/pub/plants/release-38/fasta/arabidopsis_thaliana/, $\mathrm{ftp}: / / \mathrm{ftp}$. ensemblgenomes.org/pub/plants/release-38/fasta/oryza_sativa/). To confirm the reliability of searched results, the obtained sequences were further examined based on the presence of conserved domain of CCT proteins using the InterProScan software package (version 5.25-64.0). Finally, a self-blast of protein sequences was performed to remove redundancy. Alternative splice variants were not considered. Any two protein sequences which showed a perfect match were deemed to be redundant gene pairs and the shorter sequence was removed from the potential PbCCTs list.

\section{Analysis of chromosomal locations, synteny relationships, protein properties, gene structure and conserved motifs}

The chromosome locations of PbCCTs were determined according to genome annotation data, and then plotted using MapChart software (https://www.wur.nl/en/show/Mapchart.htm). To detect gene duplication events, the Multiple Collinearity Scan toolkit (MCScan X) was applied [47]. The synteny relationship of the orthologous CCT genes between pear and Arabidopsis as well as between pear and rice was determined by using Dual Synteny Plotter software (https://github.com/CJ-Chen/TBtools). The KaKs_Calculator 2.0 was used to determine non-synonymous (Ka) and synonymous (Ks) substitution [48].

The molecular weights and theoretical isoelectric points of the PbCCT proteins were predicted by the compute pl/Mw tool in the ExPASY server (https://web.expasy.org/compute_pi/). The exon-intron structures of the PbCCTs were identified with the Gene Structure Display Server (GSDS, 
http://gsds.cbi.pku.edu.cn/) program by the alignment of cDNA sequences with the corresponding genomic sequences. The InterProScan program (http://www.ebi.ac.uk/interpro/) was used to characterize the domains and motifs of the pear CCT family.

\section{Phylogenetic analysis}

To investigate the phylogenetic relationship between pear and Arabidopsis, an unrooted neighbor-joining (NJ) trees was constructed by MEGA7 software with 1000 bootstrap replicates [49]. The numbers generated for each clade represent the bootstrap support values expressed as percentages. The same method was adopted to construct the NJ phylogenetic tree for the five subfamilies of pear CCT family.

\section{Plant materials, growth conditions and treatments}

To investigate the effects of light quality on PbCCTs gene expression, one-year-old grafted seedlings of 'Wonhwang' ( $P$. pyrifolia Nakai cv. 'Wonhwang') pear cultivar were obtained from the experimental orchard $\left(30.292^{\circ} \mathrm{N}, 114.143^{\circ} \mathrm{E}\right)$ of Research Institute of Fruit and Tea. Uniform and healthy plants were transplanted into plastic pots and cultured in an intelligent growth chamber maintained at $25^{\circ} \mathrm{C}$ and $70 \%$ relative humidity $(\mathrm{RH})$. The commercially available light source used in this study was cool-red $(\mathrm{R}) / \mathrm{blue}$ (B)/green (G) light-emitting diode (LED) panels. The distance between the lamps and pear leaves was $₫ 20$ $\mathrm{cm}$. For the R light gradient treatments, fixed $\mathrm{B}$ light $(1800 \mathrm{~lx})$ and $\mathrm{G}$ light (5000 lx) intensities were also provided. The gradual increase of $\mathrm{R}$ light included six light intensity treatments, i.e., $500 \mathrm{Ix}$ (R500), $1000 \mathrm{Ix}$ (R1000), 1500 Ix (R1500), 2000 Ix (R2000), 2500 Ix (R2500) and 3000 Ix (R3000). For the B light gradient treatments, the seedlings were sequentially incubated in six B light intensity gradients, i.e., $1000 \mathrm{~lx}$ (B1000), 1500lx (B1500), $2000 \mathrm{~lx}$ (B2000), $2500 \mathrm{~lx}$ (B2500), $3000 \mathrm{~lx}$ (B3000) and $3500 \mathrm{~lx}$ (B3500), while R light (2000 Ix) and $G$ light (5000 Ix) were fixed light intensities. The fully expanded leaves from three individual plants were defined as three biological replicates. After light irradiation, leaf samples were harvested by rapid freezing in liquid nitrogen, and stored at $-80^{\circ} \mathrm{C}$ until further use.

To test whether $P b P R R 2$ can regulate photosynthetic performance in response to varying $\mathrm{R}$ light signals, $N$. benthamiana leaves were transformed with a 35S:PbPRR2 transgene. One day after infiltration, $N$. benthamiana plants started to receive LED light treatment. The distance between the lamps and samples was $₫ 30 \mathrm{~cm}$. During the experiment, temperature was maintained at $25^{\circ} \mathrm{C}$ while $\mathrm{RH}$ was maintained at $70 \%$. A gradient of $R$ light was established, while B light ( $5000 \mathrm{~lx}$ ) and $\mathrm{G}$ light ( $1800 \mathrm{~lx}$ ) were relatively uniform. To determine the $\mathrm{R}$ light irradiance that each leaf was exposed to, light intensities (45-105 $\mu \mathrm{mol}$ $\mathrm{m}^{-2} \mathrm{~s}^{-1}$ ) were measured on the upper surface of each individual leaves using the LI-180 spectrometer (LICOR Inc., USA).

\section{Measurements of light environment among different pear tree canopy positions}

To investigate light environment among different pear tree canopy positions, sunlight spectra was measured with LI-180 spectrometer at $5 \mathrm{~cm}$ above the surface of the leaves in specific location. The adult 'Wonhwang' pear trees (ten-year-old) were grown in the experimental orchard of Research Institute of Fruit 
and Tea. The experiment was carried out in a randomized complete block design with three replications. Trees from each block were randomly selected, which represented biological replicates. Each tested tree was divided into four canopy positions, i.e., sunny side-interior part of the canopy, sunny side-exterior part of the canopy, shady side-interior part of the canopy, shady side-exterior part of the canopy. Interior and exterior parts of the canopy were approximately 0-1.0 m and more than $1.0 \mathrm{~m}$ away from the trunk, respectively. For each biological replicate of each canopy position, light quality parameters were measured on three independent positions (three technical replicates). All sunlight spectra measurements were performed every 2 hours from 08:00 to 16:00 on sunny and clear day (105 day after flowering). All data were analyzed by one-way ANOVA analyses (IBM SPSS Statistics 19 software), followed by the Duncan's multiple range tests with a significance level of $\mathrm{P}<0.01$.

\section{Expression analysis of $\mathrm{PbCCTs}$}

To investigate the expression patterns of PbCCTs in different pear tissues, the normalized RPKM (reads per kilobase per million mapped reads) values of pear CCT genes were extracted from the previously published RNA-Seq data of leaf, ovary, petal, shoot, stigma, fruit (15 days after full bloom) [50]. The results were visualized by heat map with transformed $\log _{2}(\mathrm{RPKM}+1)$ values using the 'pheatmap' $\mathrm{R}$ package (https://cran.r-project.org/web/packages/pheatmap/index.html).

In order to investigate the possible functions of the PbCCTs in the varying light quality environments, PbCCTs with higher expression level (RPKM values $>10$ ) in leaf were selected for further analysis using qRT-PCR. Total RNA was extracted from the frozen leaves using the RNAprep Pure Plant Kit (Polysaccharides \& Polyphenolics-rich) (Tiangen, China) according to the manufacturer's protocol, followed by RNA integrity examination on $1.0 \%$ agarose gels stained with ethidium bromide. First strand cDNA synthesis and qRT-PCR were performed as described previously [40]. Primer sequences for qRT-PCR analysis were designed using the Primer Premier 5 (Additional file 1). Two reference genes, i.e. PbSKD1 and $P b Y L S 8$, shown to be stably expressed in pear leaves [40], were used as internal controls to normalize the qRT-PCR data. Relative quantification was calculated according to the $\mathrm{Ct}$ method $\left(2^{-\triangle \triangle C T}\right)$. For each sample, three independent biological replicates were performed to acquire reliable results. Statistical analyses were performed as described above.

\section{Gene cloning, vector construction and transient transformation}

The cDNA sequence of PbPRR2 was amplified from 'Wonhwang' pear leaf cDNA using gene-specific primers (Additional file 1), and then cloned into the pDONR221 vector by BP reaction (Gateway, Invitrogen). Several independent clones were sequenced to confirm the correct sequence of $P b P R R 2$, then transferred by Gateway LR reaction into the destination vector pHEX2. All Gateway reactions were performed as recommended by the manufacturer (ThermoFisher Scientific, https://www.thermofisher.com). Sequence alignment was performed using Clustal X2 program (http://www.clustal.org/) and GENEDOC. pHEX-GUS, pHEX-PbPRR2 and pBIN61-p19 (the suppressor of gene silencing p19) constructs were separately introduced into the Agrobacterium tumefaciens strain 
GV3101 by electroporation method. $A$. tumefaciens cultures carring each expression vector were mixed with an equal volume of $A$. tumefaciens strain containing the pBIN61-p19, and co-infiltrated into $N$. benthamiana leaves as described in Hellens et al., (2005) [51]. Three days after infiltration, photosynthetic measurements were taken on 20 leaves per construct $(n=20)$ under different $R$ light levels, using the portable CIRAS-3 photosynthesis system (PP. Systems Inc., USA). Data were expressed as mean \pm SEM and analyzed using the IBM SPSS Statistics 19 software. The differences of the means were determined by Student's $t$-test with a significance level of $\mathrm{P}<0.001$.

\section{Results}

\section{Identification and genomic distribution of CCT family in pear}

In total, 42 PbCCTs were identified in the pear genome (Additional file 2). CCT family members were classified into COL, PRR, ZIM, TCR1 and ASML2 subfamilies, then systematically named according to their family name and sequence similarity. Our analysis revealed that COL subfamily consisted of the highest number of $C C T$ genes in pear, with 33.3\% (14 PbCOLs) of the total PbCCTs (Additional file 2). Both PRRs and ASMLs constituted the second largest subfamily with 19.0\% (8 PbPRRs and 8 PbASML2s) of the PbCCTs. The ZIM and TCR1 subfamilies were smallest, with 14.3\% (6 PbZIMs and 6 PbTCR1s) of the PbCCTs. The molecular weight of these PbCCT proteins ranged from $22.8 \mathrm{kD}$ to $93.6 \mathrm{kD}$, and their values of isoelectric point were between 4.27 and 9.45 .

PbCCTs were unevenly distributed over 15 of the 17 pear chromosomes, with no $P b C C T$ gene found on chromosome 2 and 4 (Fig. 1). Among these, chromosome 1, 3, 8 and 12 contained the fewest PbCCTs with only one member (2.4\%) on each chromosome, while chromosome 17 possessed the highest number of PbCCTs with five (11.9\%) of the 42 members. However, it should be noted, eight PbCCTs were remained on as yet unmapped scaffolds. Among all identified PbCCTs, a total of seven segmental duplication events were identified in the pear genome (Fig. 1 and Additional file 3), indicating that segmental duplication events were the major contributors to the expansion of the pear CCT family. With one exception (PbTCR5-PbTCR6), the Ka/Ks ratios of the other duplicated pairs were less than 0.26 , implying that the pear $C C T$ gene family had mainly undergone strong purifying selection (Additional file $3)$.

To further explore the synteny relationships of CCT family genes between pear and the other two representative species, Arabidopsis (dicot) and rice (monocot), we performed interspecies comparative synteny analysis in a pairwise manner (Fig. 2). A total of 109 and 35 collinear CCT gene pairs were identified in the pear/Arabidopsis and pear/rice pairs, respectively (Additional file 4). The synteny analysis also showed that multiple pear $C C T$ genes that correspond to single other plant species gene, for examples, PbCOL 1/PbCOL6/PbCOL8-At5g24930 and PbPRR1/PbPRR2/PbPRR3/PbPRR8-At2g46790 (Additional file 4). These results indicated that $C C T$ genes may have a common evolutionary ancestor among these species. Some CCT collinear gene pairs of pear/Arabidopsis were anchored to highly 
conserved collinear blocks, in which the number of syntenic gene pairs was up to 41 , whereas none of counterparts of pear/rice pairs contained more than 28 genes (Additional file 4). The high level of syntenic conservation between the pear and Arabidopsis indicated that $C C T$ genes in pear might share similar structures and functions with orthologous genes in Arabidopsis.

\section{Phylogenetic analyses of CCT genes}

To explore the phylogenetic relationship of the CCT family, an unrooted neighbor-joining phylogenetic tree was established based on the alignment of the full-length CCT protein sequences from the pear and Arabidopsis (Fig. 3). In most clades, internal nodes were supported by confidence values of at least $80 \%$, indicative of good consistency in the topology, which further corroborates the reliability of the tree. In order to test the reliability of the tree topology, protein domain architecture was used to provide additional support for the proposed phylogeny. The majority of members belonging to the same phylogenetic group exhibited common motif compositions (Fig. 4D). For example, TIFY and C2C2-GATA zinc-finger domains are specifically shared by ZIM subfamily. Presence of the Pseudo-receiver domain is also clade dependent in the PRR subfamily. The conserved intron/exon structural characteristics also supported the fine structure of the phylogenetic trees. For example, all the coding sequences of the PbPRRs were interrupted by 5 or 7 introns, while the TCR 1 subfamily contained no more than two introns.

According to the classification criteria of CCT family in Arabidopsis, PbCCTs were classified into seven major clades, named Clade I-VII (Fig. 3). 14 PbCOLs were categorized into three clades, with wellsupported bootstrap values: five PbCOLs in Clade I, four PbCOLs in Clade II, and five PbCOLs in Clade III (Fig. 3 and Fig. 4A). Clade I comprised of five PbCOLs (PbCOL 1/4/5/8/9), featuring a conserved CCT domain with two upstream zinc-finger B-box domains (Fig. 4D). PbCOL members ( $P b C O L 2 / 3 / 6 / 7)$ in Clade II exhibited one B-box domain and a CCT domain. The gene structures of Clade I and II were highly conserved, containing two exons and one intron (Fig. 4C). The PbCOL homologs (PbCOL 10-PbCOL 14) were clustered with the Clade III of Arabidopsis COL subfamily that possesses a normal B-box domain, a second divergent B-box domain and a CCT domain. We found that this phylogenic classification of $P b C O L s$ in Clade I and Clade II was the same as the classification of Arabidopsis COL subfamily based on the difference of the B-box domain (Fig. 3 and Fig. 4D). However, PbCOL 10, PbCOL 11 and PbCOL 12 in Clade III contained only one or no B-box domain. These patterns suggest that the corresponding genes may have lost the B-box type zinc finger domain.

The PRR subfamily was further divided into three main subgroups based on their phylogenetic relationship, named as Clade IVa, Clade IVb and Clade IVc (Fig. 3). Four members (PbPRR1/4/5/6) in Clade IVa and two members (PbPRR2/3) in Clade IVb were highly conserved, containing eight exons and seven introns (Fig. 4C). Clade IVc included two pear PRR genes (PbPRR7/8) that clustered with AtPRR1 (AtTOC1) gene from the same branch (Fig. 3).

The TCR subfamily could be divided into two subgroups, i.e. Clade Va and Clade Vb (Fig. 3). For the Clade Va, PbTCR1 and PbTCR4 were clustered with two AtTCR genes (At5g57180 and At4g25990). Clade Vb 
contained four pear members (PbTCR2/3/5/6), which were clustered with two AtTCR genes (AtTCR1 and At5g14370).

All pear ZIM genes were also divided into two subgroups (Fig. 3). In detail, Clade Vla was comprised of three pear PbZIM members ( $P b Z M L 4 / 5 / 6$ ), which clustered with Arabidopsis ZIM subfamily genes (AtZIM, AtZML1 and AtZML2), while three pear ZIM homologs ( $P b Z M L 1 / 2 / 3)$ were identified as a distinct subgroup (Clade $\mathrm{Vlb}$ ) that had no counterpart in Arabidopsis.

Pear ASML2 members were classified into four subgroups and were characterized by only conserved CCT domain (Fig. 3 and Fig. 4D). Clade Vlla and Clade Vllb were divided from the same branch, and contained $P b A S M L 3 / 4$ and $P b A S M L 7 / 8$, respectively. PbASML $1 / 2$ were clustered to Clade VIlc and $P b A S M L 5 / 6$ belonged to Clade VIId.

\section{Expression profiles of CCT genes in different tissues and under varying light signals environments}

To investigate the tissue expression profiles of the PbCCTs in pear, we analyzed their transcript levels based on a publically available RNA-seq data of different tissues, including leaf, ovary, petal, shoot, stigma and fruit (Fig. 4B). In general, the candidate PbCCTs showed variation in tissue expression patterns. Many PbPRRs and PbCOLs exhibited high transcript abundance level in all six tissues, whereas most $P b A S M L s$ were expressed at relatively lower levels in multiple tissues. On the other hand, several PbCCTs exhibited tissue-specific expression. For example, PbCOL6 and PbTCR3 were mainly expressed in leaf, whereas $P b C O L 9$ showed the highest transcript abundance in the petal. Some duplicated gene pairs also showed divergent transcript levels. For instance, $P b Z M L 3$ showed very low expression in six different tissues; whereas its duplicated gene, $P b Z M L 2$, was highly expressed in all tested tissues. These results suggest that duplicated genes may evolve to have diverse functions.

We investigate the environmental light spectrum changes among different pear tree canopy positions. Compared with the exterior part of the canopy, the levels of R and B light decreased significantly in the interior part of the canopy, indicating that intensity changes of light quality are important signatures in fruit orchards (Additional file 5). Partial genes from CCT family have showed to regulate growth and development by responding to light signals; therefore, the response of the PbCCTs induction to light quality treatments was further characterized by qRT-PCR. Overall, most of selected PbCCTs showed highly diverse expression patterns under the enhancement of R/B light radiation (Fig. 5 and Fig. 6). These results suggested that they are sensitive to light quality signals, thus, intriguing different responses according to the external light conditions. Under the $\mathrm{R}$ light treatments, the expression of three genes (PbCOL6, PbTCR1, PbTCR2) reached a peak at the early stage (R1000), and then down-regulated during subsequent increased exposure to R light (Fig. 5). Additionally, PbCOL11 and PbTCR3 presented an increasing trend with the increasing $\mathrm{R}$ light process. It was found that one homeologous pair (PbPPR2 and $P b P P R 3)$ displayed strong rhythmic expression patterns, suggesting that these genes could respond to $\mathrm{R}$ light changes during their regulation on pear growth and development. Noteworthy, compared with the control (R500), the abundance of PbPRR2 and PbTCR3 transcripts dramatically increased more than 13.5-fold at R2500 stage and 107.6-fold at R3000 stage, respectively. Meanwhile, we also analyzed the 
expression pattern of the PbCCTs in the B light treatment (Fig. 6). Among them, the transcript levels of four genes (PbPPR2, PbPRR3, PbTCR1 and PbTCR3) were induced by enhanced $\mathrm{B}$ light, some of which decreased markedly at the highest abundance (B3500). Notably, the relative expression level of $P b P P R 2$ approximately increased 208.9-fold at B3000 stage relative to control and that of $P b T C R 3$ approximately increased 172.0-fold at B3500 stage. In addition, two PbCCTs (PbCOL5 and PbTCR4) showed the decreased expression levels in response to progressively increasing $B$ light signals.

\section{PbPRR2 is a candidate for negatively regulating photosynthetic performance}

Combining the previous transcriptomic study, present bioinformatics analysis and expression analysis [41], PbPRR2 (LOC103943360), a close homologue of Arabidopsis circadian clock gene AtPRR5, was chosen as a strong candidate for functional verification. Because AtPRR5 are implicated photomorphogenesis in $\mathrm{R}$ light that is considered as the most efficient wavelength for driving photosynthesis [52-55], these facts prompted us to examine the photosynthetic performance of plants overexpressing PbPRR2 under a broad range of $\mathrm{R}$ light intensity. PbPRR2 has a 2013-bp open reading frame and encodes a protein of 670 amino acids (GenBank accession number: MZ826141). The amino acid sequences encoded by PbPRR2 and AtPRR5 (AT5G24470) - the ortholog of PbPRR2 from Arabidopsis - are $40.12 \%$ identical (Additional file 6). PbPRR2 protein featured a PR domain at the $\mathrm{N}$ terminus and a CCT motif at the $\mathrm{C}$ terminus.

To further test the role of $P b P R R 2$ in the regulation of photosynthetic properties under the changing $\mathrm{R}$ light signals, $P b P R R 2$ was transiently over-expressed in $N$. benthamiana leaves and compared with the control leaves. Fluctuating profiles of net photosynthetic rate, stomatal conductance and internal $\mathrm{CO}_{2}$ were observed in both PbPRR2 infiltrated and control leaves with the increasing $\mathrm{R}$ light intensity (Fig. 7). However, significantly reduced levels of these photosynthetic parameters were observed in leaves inoculated with the pHEX2-PbPRR2 construct compared to the pHEX2-GUS control. These observations indicate that $P B P R R 2$ may suppress the red light-dependent enhancement of photosynthetic performance.

\section{Discussion}

Photosynthetic performance is an important agricultural trait vital to the adaptation of horticultural crops to ambient light conditions [56]. Increasing evidences suggest that $C C T$ genes play important roles in light-regulated developmental processes $[10,45,53,57,58]$. Understanding the structural characteristics of pear CCT family genes and their specific responses to different light environments will help to find some important candidates that may be involved in canopy light signal regulation. Here, we performed a comprehensive analysis of the PbCCT family genes. A total of $42 \mathrm{PbCCT}$ genes were identified from pear genome, accounting for $0.098 \%$ of all predicted protein-coding genes (42,812 genes) [46], which was less than that in Arabidopsis thaliana (36 AtCCT genes out of the 25,498 predicted genes, accounting for $0.141 \%$ ) [7,59], but higher than that in Oryza sativa (41 OsCCT genes out of the 53,398 predicted gene, accounting for $0.076 \%)[8,60,61]$. These results suggested that the $C C T$ family members may not be 
directly related to the genome sizes in different plants, since the sizes (512 Mb) of the pear genomes are larger than that of rice (403 Mb) and even about fourfold of that of Arabidopsis $(125 \mathrm{Mb})[46,59,60]$. In the process of genome evolution, gene duplication has generally been viewed as a necessary source of gene family expansion $[62,63]$. In this study, none of the PbCCTs were located in tandem, and seven segmental duplication events were identified in the PbCCTs, suggesting that the segmental duplication was the main contributor to the expansion of PbCCTs; similar results were also observed for other gene families in pear [64-67]. After gene duplication, some duplicates may undergo functional divergence. The $\mathrm{Ka} / \mathrm{Ks}$ ratios of $\mathrm{PbCCT}$ duplication pair indicated that purifying selection was a major force driving the evolution of new functions for PbCCTs.

Phylogenetic analysis showed that most of the CCT family clades contained both pear and Arabidopsis proteins, suggesting that the two species display relatively conserved evolution (Fig. 3). Gene expression patterns of $P b C C T s$ in response to varying light quality could provide important clues regarding gene functions during light response process. For instance, two PbCCTs ( $P b C O L 5$ and $P b C O L 7$ ), showed progressively decreasing expression levels with increasing R light intensity (Fig. 5). AtCO1, an Arabidopsis ortholog of PbCOL5 (Fig. 3), is a key player in the induction of flowering [18]. AtCO protein was degraded under R light by Phytochrome B [68]. Our result showed that shaded light had lower intensity of R/B light (Additional file 5). These findings suggest that increased $\mathrm{PbCOL} 5$ expression induces early flowering in response to the lower $\mathrm{R}$ light level to which pear shoots are exposed when shaded by neighboring vegetation. Also the expression of AtCOL 7, which is a homolog gene of PbCOL 7 in Arabidopsis (Fig. 3), was rapidly down-regulated in response to high R:FR (Red:far-red light) [58]. Overexpression of AtCOL7 was shown to enhance branching number under high R:FR conditions. It would be interesting to determine if $P b C O L 7$ could increase branching by perceiving decreasing $\mathrm{R}$ light signaling.

In Arabidopsis, the AtCOL13 was shown to act as a positive regulator of $\mathrm{R}$ light mediated inhibition of hypocotyl elongation [69]. The transcription level of PbCOL11, a homolog gene of AtCOL 13, was upregulated robustly under the enhanced $\mathrm{R}$ light environment (Fig. 3 and Fig. 5), implying that PbCOL 14 might act in response to $\mathrm{R}$ light signaling to regulate photomorphogenesis in pear.

Our results provided evidence indicating that $P b P R R 2$ may have a novel role in the regulation of photosynthetic performance under varying light signals environment. Our phylogenetic analysis showed that the homeologous pair (PbPRR2/PbPRR3) clustered together with Arabidopsis AtPRR5 (Fig. 3). The functions of AtPRR5 were implicated in the mechanisms underlying the control of flowering time and photomorphogenesis, as well as the circadian rhythm [52, 70]. Moreover, AtPRR5-ox plants are shown to be highly sensitive to continuous $\mathrm{R}$ light, and also seemed to be slightly hypersensitive to $B$ light [70]. "Red or far-red light signaling pathway" was found to be an enriched category in AtPRR5 direct-targets [26]. Here, we noticed that the expression of $P b P R R 2$ and $P b P R R 3$ showed robust free-running rhythms under continuously increasing $\mathrm{R}$ light (Fig. 5). Our previous study indicated that these two genes, which involved in 'Circadian rhythm-plant' pathway, were strongly correlation with photosynthetic performance [41]. A key piece of evidence supporting the role of $P b P R R 2$ in light response is that it resulted in inhibition of photosynthetic performance as compared with those of the control plants (Fig. 7). Therefore, 
PbPRR2 (together with PbPRR3) should be taken into consideration for a better understanding of the molecular links between circadian rhythms and light signaling-controlled photosynthetic performance.

\section{Conclusions}

In short, a total of 42 PbCCTs were identified in pear and divided into five subfamilies, as supported by phylogenetic relationship, intron-exon structures and conserved motifs. Expression analysis indicated that $\mathrm{PbCCT}$ family might have diverse functions and some members were sensitive to light induction, indicating that PbCCT genes were involved in light environmental adaptation. One CCT gene named $P b P R R 2$ was indicated to be a negative regulator of photosynthetic performance under the enhanced $\mathrm{R}$ light environment. All the results presented in this study provide comprehensive information on the CCT family in pear and lay a foundation for further research on the function of $P b C C T$ gene.

\section{Abbreviations}

Ka: non-synonymous substitution; Ks: synonymous substitution; NJ: neighbor-joining; RH: relative humidity; R: red; B: blue; G: green; LED: light-emitting diode; RPKM: reads per kilobase per million mapped reads; FR: far-red; R500/1000/1500/2000/2500/3000: six red light intensity gradients, i.e., 500 lx, 1000 lx, $1500 \mathrm{~lx}, 2000 \mathrm{~lx}, 2500 \mathrm{~lx}$ and $3000 \mathrm{~lx}$; B1000/1500/2000/2500/3000/3500: six blue light intensity gradients, i.e., $1000 \mathrm{~lx}, 1500 \mathrm{~lx}, 2000 \mathrm{~lx}, 2500 \mathrm{~lx}, 3000 \mathrm{~lx}$ and $3500 \mathrm{~lx}$.

\section{Declarations}

\section{Ethics approval and consent to participate}

Not applicable.

\section{Consent for publication}

Not applicable.

\section{Availability of data and materials}

All data generated or analyzed during this study are included in this published article and its supplementary information files.

\section{Competing interests}

The authors declare that they have no competing interests.

\section{Funding}

This work was financially supported by the

National Key R\&D Program of China (no. 2018YFD1000200), the 
China Agriculture Research System of MOF and MARA (no. CARS-28-11), the Major Program of Hubei Agricultural Science and Technology Innovation Center (no. 2020-620-000-002-05), and the National Natural Science Foundation of China (nos. 31801819). The funders had no role in study design, data collection and analysis, decision to publish, or preparation of the manuscript.

\section{Authors' contributions}

ZL and TW conceived and designed the research. ZL, JLL and LA performed the experiments. ZL, $L Y, Y S C$ and XSN analyzed the data. ZL wrote the paper. ZQQ revised the paper. All authors have read and approved the manuscript for publication.

\section{Acknowledgements}

Not applicable.

\section{References}

1. Li Y, Xu M. CCT family genes in cereal crops: A current overview. Crop J. 2017;5:449-458.

2. Mengarelli DA, Zanor MI. Genome-wide characterization and analysis of the CCT motif family genes in soybean (Glycine max). Planta. 2021;253:15.

3. Hotta CT, Xu X, Xie Q, Dodd AN, Johnson CH, Webb AAR. Are there multiple circadian clocks in plants? Plant Signal. Behav. 2008;3:342-344.

4. Gangappa SN, Botto JF. The BBX family of plant transcription factors. Trends Plant Sci. 2014;19:460-470.

5. Kaewphalug W, Huehne PS, Sriboonlert A. Characterization of a CONSTANS-like gene from pigeon orchid (Dendrobium crumenatum Swartz) and its expression under different photoperiod conditions. Hortic. J. 2017;86:252-262.

6. Masaki T, Tsukagoshi H, Mitsui N, Nishii T, Hattori T, Morikami A, et al. Activation tagging of a gene for a protein with novel class of CCT-domain activates expression of a subset of sugar-inducible genes in Arabidopsis thaliana. Plant J. 2005;43:142-152.

7. Iwata Y, Yamada T, Koizumi N. Transcriptional regulation of an Arabidopsis gene encoding a CCT domain-containing protein during endoplasmic reticulum stress. Plant Biotechnol. 2008;25:397-402.

8. Cockram J, Thiel T, Steuernagel B, Stein N, Taudien S, Bailey PC, et al. Genome dynamics explain the evolution of flowering time CCT domain gene families in the Poaceae. PLoS ONE. 2012;7:e45307.

9. Zheng X, Li X, Ge C, Zhang J, Shi M, Chen J, et al. Characterization of the CCT family and analysis of gene expression in Aegilops tauschii. PLoS ONE. 2017;12:e0189333.

10. Jin M, Liu X, Jia W, Liu H, Li W, Peng Y, et al. ZmCOL3, a CCT gene represses flowering in maize by interfering circadian clock and activating expression of ZmCCT. J. Integer. Plant Biol. 2018;60:465480 . 
11. Ma L, Yi D, Yang J, Liu X, Pang Y. Genome-wide identification, expression analysis and functional study of CCT gene family in Medicago truncatula. Plants. 2020;9:513.

12. Liu HY, Zhou XC, Li QP, Wang L, Xing YZ. CCT domain-containing genes in cereal crops: flowering time and beyond. Theor. Appl. Genet. 2020;133:1385-1396.

13. Kurokura T, Samad S, Koskela E, Mouhu K, Hytönen T. Fragaria vesca CONSTANS controls photoperiodic flowering and vegetative development. J. Exp. Bot. 2017;68:4839-4850.

14. Li YF, Zhao YQ, Zhang M, Jia GX, Zaccai M. Functional and evolutionary characterization of the CONSTANS-like family in Lilium× formolongi. Plant Cell Physiol. 2018;59:1874-1888.

15. Song N, Xu Z, Wang J, Qin Q, Jiang H, Si W, et al. Genome-wide analysis of maize CONSTANS-LIKE gene family and expression profiling under light/dark and abscisic acid treatment. Gene. 2018;673:111.

16. Xu D, Jiang Y, Li J, Holm M, Deng XW. The B-box domain protein BBX21 promotes photomorphogenesis, Plant Physiol. 2018;176:2365-2375.

17. Li J, Gao K, Yang X, Khan WU, Guo B, Guo T, et al. Identification and characterization of the CONSTANS-like gene family and its expression profiling under light treatment in Populus. Int. J. Bio. Macromolecules. 2020;161:999-1010.

18. Suárez-López P, Wheatley K, Robson F, Onouchi H, Valverde F, Coupland G. CONSTANS mediates between the circadian clock and the control of flowering in Arabidopsis. Nature. 2001;410:11161120.

19. An H, Roussot C, Suárez-López P, Corbesier L, Vincent C, Piñeiro M, et al. CONSTANS acts in the phloem to regulate a systemic signal that induces photoperiodic flowering of Arabidopsis. Development. 2004;131:3615-3626.

20. Yoo SK, Chung KS, Kim J, Lee JH, Hong SM, Yoo SJ, et al. CONSTANS activates SUPPRESSOR OF OVEREXPRESSION OF CONSTANS 1 through FLOWERING LOCUS T to promote flowering in Arabidopsis. Plant Physiol. 2005;139:770-778.

21. Min JH, Chung JS, Lee KH, Kim CS. The CONSTANS-like 4 transcription factor, AtCOL4, positively regulates abiotic stress tolerance through an abscisic acid-dependent manner in Arabidopsis. J. Integr. Plant. Biol. 2015;57:313-324.

22. Liu J, Shen J, Xu Y, Li X, Xiao J, Xiong L. Ghd2, a CONSTANSlike gene, confers drought sensitivity through regulation of senescence in rice. J. Exp. Bot. 2016;67:5785-5798.

23. Muntha ST, Zhang L, Zhou Y, Zhao X, Hu Z, Yang J, et al. Phytochrome A signal transduction 1 and CONSTANS-LIKE 13 coordinately orchestrate shoot branching and flowering in leafy Brassica juncea. Plant Biotechnol. J. 2018;17:1333-1343.

24. Ohmiya A, Oda-Yamamizo C, Kishimoto S. Overexpression of CONSTANS-like 16 enhances chlorophyll accumulation in petunia corollas. Plant Sci. 2019;280:90-96.

25. Cao D, Lin Z, Huang L, Damaris RN, Li M, Yang P. A CONSTANS-LIKE gene of Nelumbo nucifera could promote potato tuberization. Planta. 2021;253:65. 
26. Nakamichi N, Kiba T, Kamioka M, Suzuki T, Yamashino T, Higashiyama T, et al. Transcriptional repressor PRR5 directly regulates clock-output pathways. Proc. Natl. Acad. Sci. U.S.A. 2012;109:17123-17128.

27. Farré EM, Liu T. The PRR family of transcriptional regulators reflects the complexity and evolution of plant circadian clocks. Curr. Opin. Plant Biol. 2013;16:621-629.

28. Liu TL, Newton L, Liu MJ, Shiu SH, Farré EM. A G-box-like motif is necessary for transcriptional regulation by circadian Pseudo-Response Regulators in Arabidopsis. Plant Physiol. 2016;170:528539.

29. Majeed N, Panigrahi KCS, Sukla LB, John R, Panigrapy M. Regulatory mechanisms across networks of the circadian clock and senescence pathways. J. Plant Biochem. Biotechnol. 2020;29:665-674.

30. Liu T, Carlsson J, Takeuchi T, Newton L, Farré EM. Direct regulation of abiotic responses by the Arabidopsis circadian clock component PRR7. Plant J. 2013;76:101-114.

31. Zhang L, You J, Chan Z. Identification and characterization of TIFY family genes in Brachypodium distachyon. J. Plant Res. 2015;128:995-1005.

32. Saha G, Park JI, Kayum MA, Nou IS. A genome-wide analysis reveals stress and hormone responsive patterns of TIFY family genes in Brassica rapa. Front. Plant Sci. 2016;7:936.

33. Shikata M, Takemura M, Yokota A, Kohchi T. Arabidopsis ZIM, a plant-specific GATA factor, can function as a transcriptional activator. Biosci. Biotechnol. Biochem. 2003;67:2495-2497.

34. Shaikhali J, Barajas-Lopéz JD, Ötvös K, Kremnev D, Garcia AS, Srivastava V, et al. The CRYPTOCHROME1-dependent response to excess light is mediated through the transcriptional activators ZINC FINGER PROTEIN EXPRESSED IN INFLORESCENCE MERISTEM LIKE1 and ZML2 in Arabidopsis. Plant Cell. 2012;24:3009-3025.

35. Vélez-Bermúdez IC, Salazar-Henao JE, Fornalé S, López-Vidriero I, Franco-Zorrilla JM, Grotewold E, et al. A MYB/ZML complex regulates wound-induced lignin genes in maize. Plant Cell. 2015;27:32453259.

36. Masaki T, Tsukagoshi H, Mitsui N, Nishii T, Hattori T, Morikami A, et al. Activation tagging of a gene for a protein with novel class of CCT-domain activates expression of a subset of sugar-inducible genes in Arabidopsis thaliana. Plant J. 2005;43:142-152.

37. Valim HF, McGale E, Yon F, Halitschke R, Fragoso V, Schuman MC, et al. The clock gene TOC1 in shoots, not roots, determines fitness of Nicotiana attenuata under drought. Plant Physiol. 2019;181:305-318.

38. Lyu G, Li D, Li S. Bioinformatics analysis of BBX family genes and its response to UV-B in Arabidopsis thaliana. Plant Signal. Behav. 2020;15:9.

39. Wang S, Zhang C, Zhao J, Li R, Lv J. Expression analysis of four pseudo-response regulator (PRR) genes in Chrysanthemum morifolium under different photoperiods. Peer J. 2019;7:e6420.

40. Liu Z, Cheng K, Qin Z, Wu T, Li X, Tu J, et al. Selection and validation of suitable reference genes for qRT-PCR analysis in pear leaf tissues under distinct training systems. Plos ONE. 2018;13:e0202472. 
41. Liu Z, An L, Lin S, Wu T, Li X, Tu J, et al. Comparative physiological and anscriptomic analysis of pear leaves under distinct training systems. Sci Rep. 2020;10:18892.

42. Wang Q, Xie W, Xing H, Yan J, Meng X, Li X, et al. Genetic architecture of natural variation in rice chlorophyll content revealed by a genome-wide association study. Mol. Plant 2015;8:946-957.

43. Morita R, Sugino M, Hatanaka T, Misoo S, Fukayama H. $\mathrm{CO}_{2}$-responsive CONSTANS, CONSTANS-like, and time of Chlorophyll $a / b$ binding protein expression 1 protein is a positive regulator of starch synthesis in vegetative organs of rice. Plant Physiol. 2015;167:1321-1331.

44. Morita R, Inoue K, Ikeda Kl, Hatanaka T, Misoo S, Fukayama H. Starch content in leaf sheath controlled by $\mathrm{CO}_{2}$-responsive $\mathrm{CCT}$ protein is a potential determinant of photosynthetic capacity in rice. Plant Cell Physiol. 2016;57:2334-2341.

45. Heng $Y$, Jiang $Y$, Zhao $X$, Zhou $H$, Wang $X$, Deng XW, et al. BBX4, a phyB-interacting and modulated regulator, directly interacts with PIF3 to fine tune red light-mediated photomorphogenesis. Proc. Natl. Acad. Sci. U.S.A. 2019;116:26049-26056.

46. Wu J, Wang Z, Shi Z, Zhang S, Ming R, Zhu S, et al. The genome of the pear (Pyrus bretschneideri Rehd.). Genome Res. 2013;23:396-408.

47. Wang Y, Tang H, DeBarry JD, Tan X, Li J, Wang X, et al. MCScan X: a toolkit for detection and evolutionary analysis of gene synteny and collinearity, Nucleic Acids Res. 2012;40:e49.

48. Wang D, Zhang Y, Zhang Z, Zhu J, Yu J. KaKs_Calculator 2.0: a toolkit incorporating gamma-series methods and sliding window strategies. Genomics Proteomics Bioinformatics. 2010;8:77-80.

49. Kumar S, Stecher G, Tamura K. MEGA7: molecular evolutionary genetics analysis version 7.0 for bigger datasets. Mol Biol Evol. 2016;33:1870-1874.

50. Zhang S, Ma M, Zhang H, Zhang S, Qian M, Zhang Z, et al. Genome-wide analysis of polygalacturonase gene family from pear genome and identification of the member involved in pear softening. BMC Plant Biol. 2019;19:587.

51. Hellens RP, Allan AC, Friel EN, Bolitho K, Grafton K, Templeton MD, et al. Transient expression vectors for functional genomics, quantification of promoter activity and RNA silencing in plants. Plant Methods. 2005;1:13.

52. Yamamoto Y, Sato E, Shimizu T, Nakamich N, Sato S, Kato T, et al. Comparative genetic studies on the APRR5 and APRR7 genes belonging to the APRR1/TOC1 quintet implicated in circadian rhythm, control of flowering time, and early photomorphogenesis. Plant Cell Physiol. 2003;44:1119-1130.

53. Kato T, Murakami M, Nakamura Y, Ito S, Nakamichi N, Yamashino T, et al. Mutants of circadianassociated $P R R$ genes display a novel and visible phenotype as to light responses during deetiolation of Arabidopsis thaliana Seedlings. Biosci. Biotechnol. Biochem. 2007;71:834-839.

54. Izzo LG, Mele BH, Vitale L, Vitale E, Arena $\mathrm{C}$. The role of monochromatic red and blue light in tomato early photomorphogenesis and photosynthetic traits. Environ. Exp. Bot. 2020;179:104195.

55. Liu J, van lersel MW. Photosynthetic physiology of blue, green, and red light: light intensity effects and underlying mechanisms. Front. Plant Sci. 2021;12:619987. 
56. Fu QS, Zhao B, Wang YJ, Ren S, Guo YD. Stomatal development and associated photosynthetic performance of capsicum in response to differential light availabilities. Photosynthetica. 2010;48:189-198.

57. Lee YS, Jeong DH, Lee DY, Yi J, Ryu CH, Kim SL, et al. OsCOL4 is a constitutive flowering repressor upstream of Ehd1 and downstream of OsphyB. Plant J. 2010;63:18-30.

58. Wang $\mathrm{H}$, Zhang Z, Li H, Zhao X, Liu X, Ortiz M, et al. CONSTANS-LIKE 7 regulates branching and shade avoidance response in Arabidopsis. J. Exp. Bot. 2013;64:1017-1024.

59. The Arabidopsis Genome Initiative. Analysis of the genome sequence of the flowering plant Arabidopsis thaliana. Nature. 2000;408:796-815.

60. Yu J, Hu S, Wang J, Wong GKS, Li S, Liu B, et al. A draft sequence of the rice genome (Oryza sativa L. ssp. indica). Science. 2002;296:79-92.

61. Zhang J, Hu Y, Xu LH, He Q, Fan XW, Xing YZ. The CCT domain-containing gene family has large impacts on heading date, regional adaptation, and grain yield in rice. J. Integer. Agric. 2017;16:26862697.

62. Su W, Ren Y, Wang D, Su Y, Feng J, Zhang C, et al. The alcohol dehydrogenase gene family in sugarcane and its involvement in cold stress regulation. BMC Genomics. 2020;21:521.

63. Wang J, Wang Z, Jia C, Miao H, Zhang J, Liu J, et al. Genome-wide identification and transcript analysis of TCP gene family in Banana (Musa acuminata L.). Biochem. Genet. 2021; https://doi.org/10.1007/s10528-021-10100-8.

64. Cao Y, Han Y, Li D, Lin Y, Cai Y. MYB transcription factors in Chinese pear (Pyrus bretschneideri Rehd.): genome-wide identification, classification, and expression profiling during fruit development. Front. Plant Sci. 2016;7:577.

65. Abdullah M, Cao Y, Cheng X, Meng D, Chen Y, Shakoor A, et al. The sucrose synthase gene family in Chinese pear (Pyrus bretschneideri Rehd.): structure, expression, and evolution. Molecules. 2018;23:1144.

66. Wang L, Wang L, Zhang Z, Ma M, Wang R, Qian M. et al. Genome-wide identification and comparative analysis of the superoxide dismutase gene family in pear and their functions during fruit ripening. Postharvest Biol. Technol. 2018;143:68-77.

67. Wang Y, Lü J, Chen D, Zhang J, Qi KJ, Cheng R, et al. Genome-wide identification, evolution, and expression analysis of the KT/HAK/KUP family in pear. Genome. 2018;61:755-765.

68. Valverde F, Mouradov A, Soppe W, Ravenscroft D, Samach A, Coupland G. Photoreceptor regulation of CONSTANS protein in photoperiodic flowering. Science. 2004;303:1003-1006.

69. Liu B, Long H, Yan J, Ye L, Zhang Q, Chen H, et al. A HY5-COL3-COL13 regulatory chain for controlling hypocotyl elongation in Arabidopsis. Plant Cell Environ. 2021;44:130-142.

70. Sato E, Nakamichi N, Yamashino T, Mizuno T. Aberrant expression of the Arabidopsis circadianregulated APRR5 gene belonging to the APRR1/TOC1 quintet results in early flowering and hypersensitiveness to light in early photomorphogenesis. Plant Cell Physiol. 2002;43:1374-1385. 
Figures

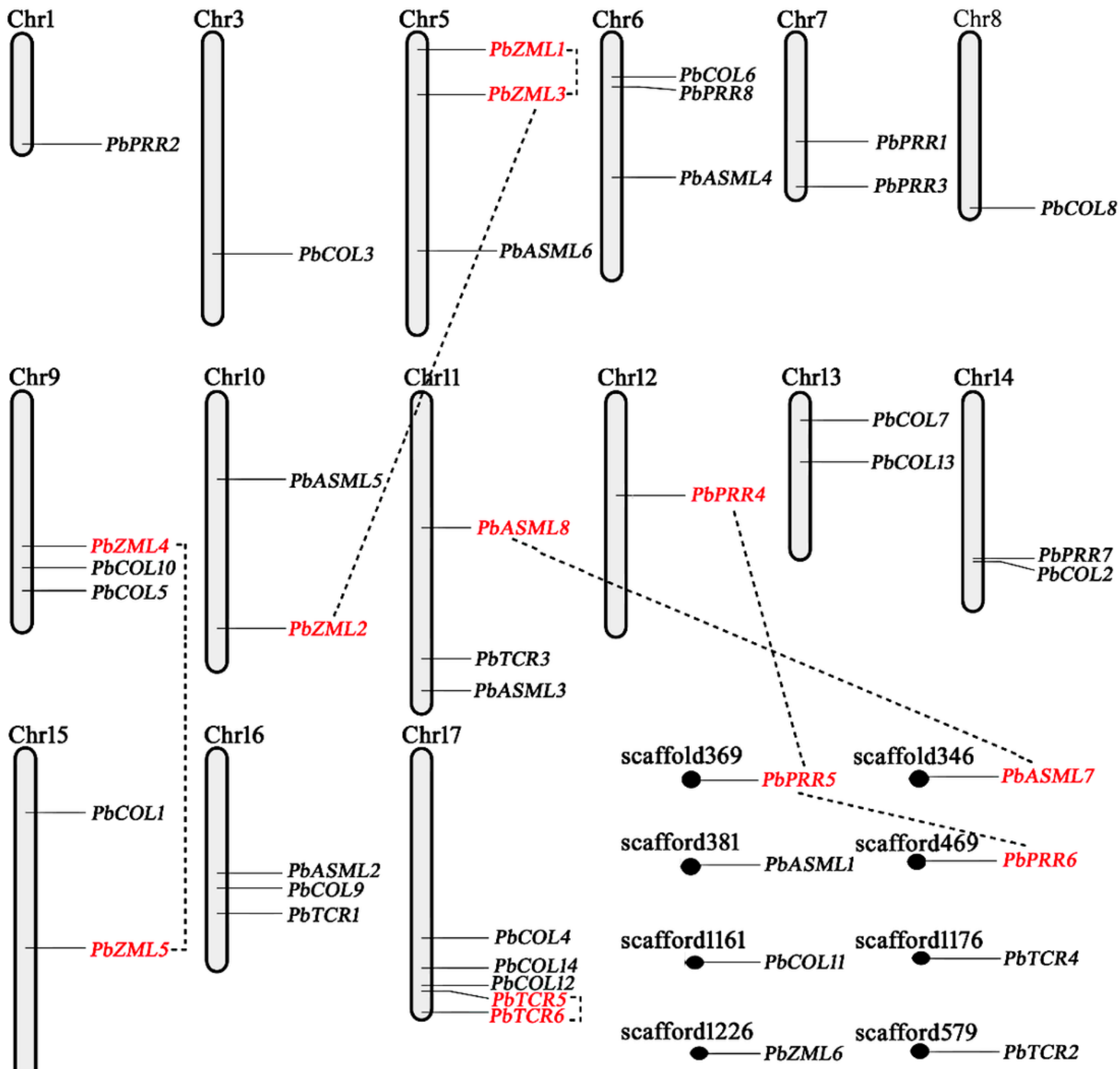

Figure 1

Chromosomal locations and regional duplication of pear CCT genes. The chromosomal position of each CCT gene was mapped to the pear genome. The chromosome number is indicated at the top of each chromosome. Duplicated gene pairs are linked by dotted lines. 


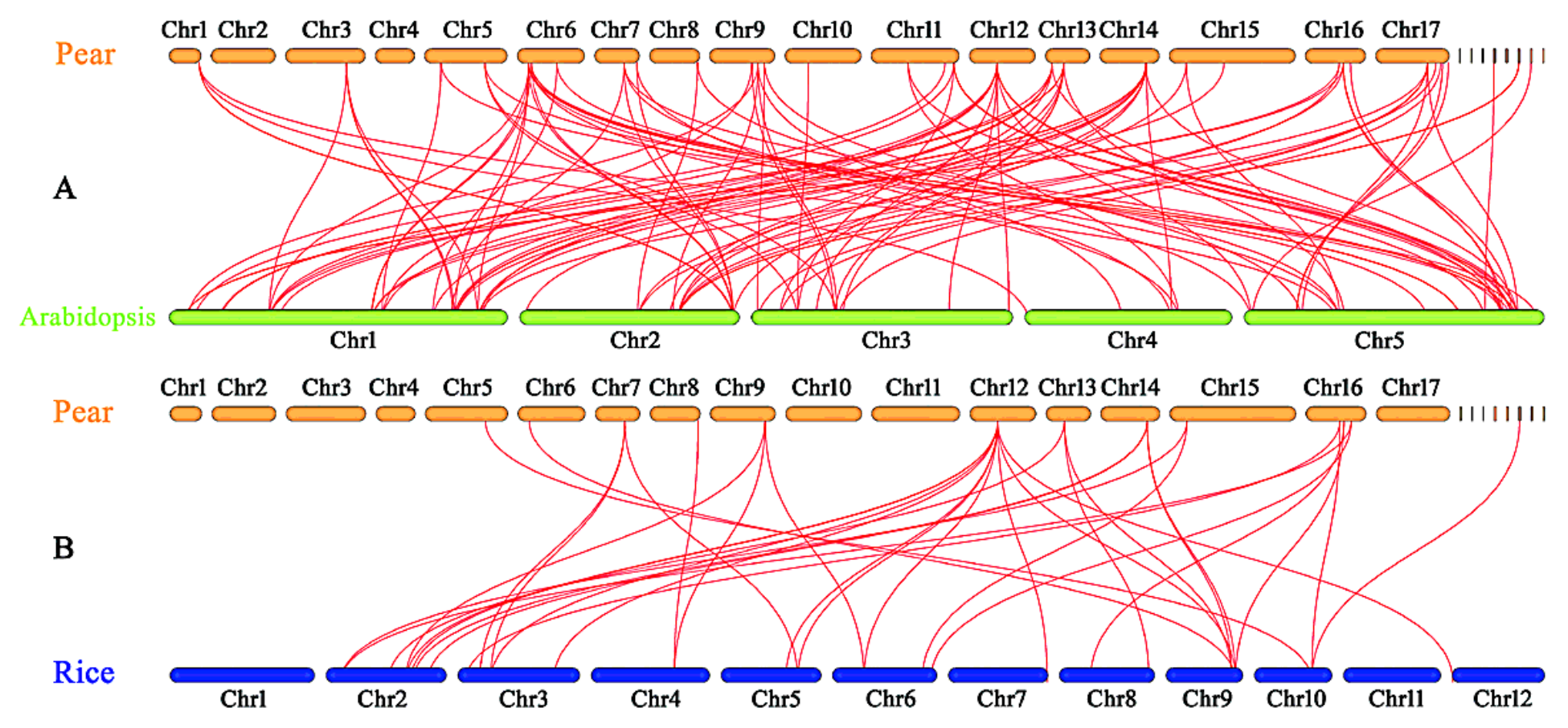

Figure 2

Gene duplication and synteny analysis of the CCT genes between pear and Arabidopsis/rice. Gray lines in the background indicate the collinear blocks within pear/Arabidopsis genomes $(A)$ and pear/rice genomes (B), respectively. The red lines highlight the syntenic CCT gene pairs. 


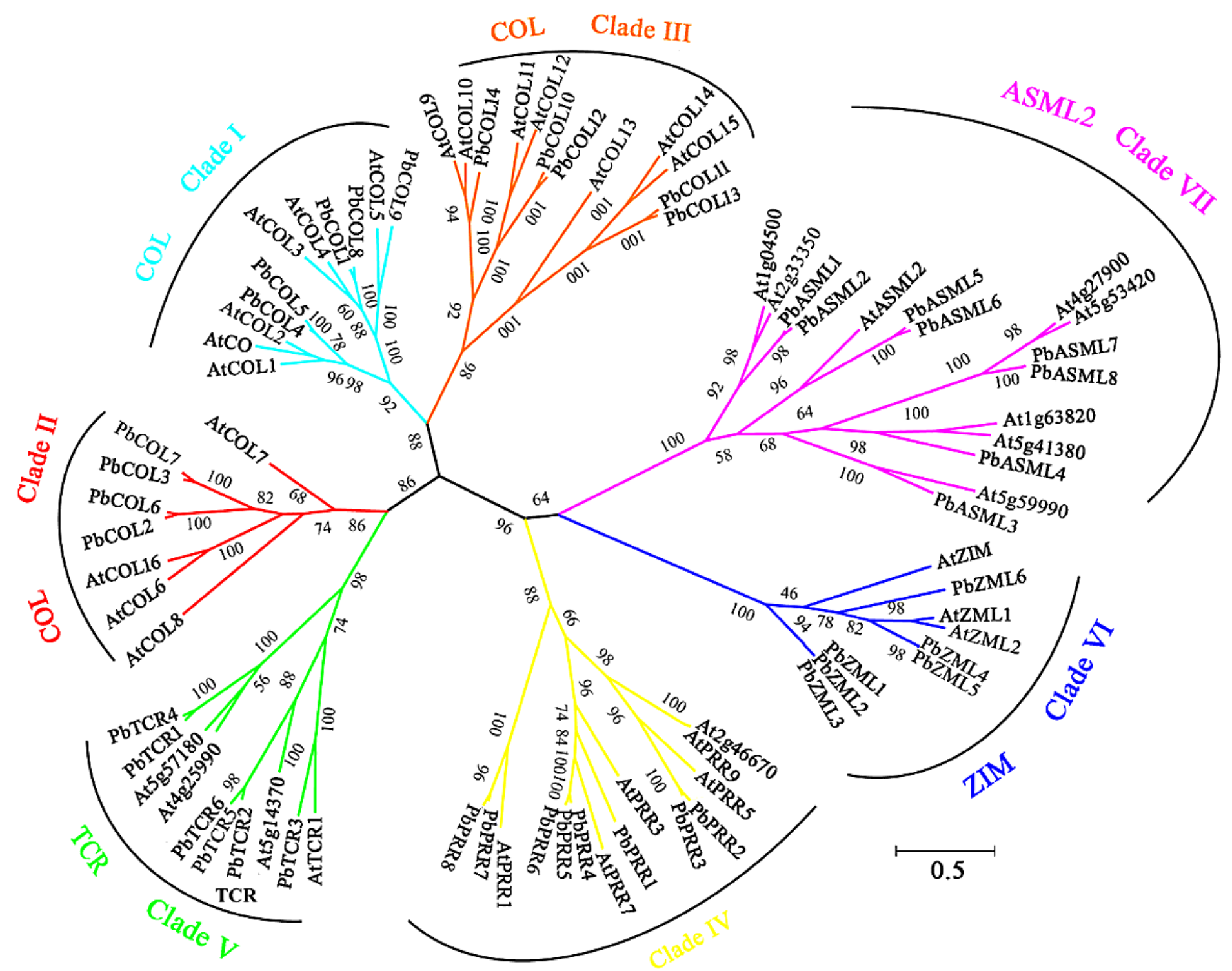

Figure 3

Phylogenetic relationships and subfamily designations in CCT proteins from pear and Arabidopsis based on the neighbor-joining method. The reliability of the predicted tree was tested by bootstrapping with 1000 replicates. The percentage of neighbor-joining bootstrap replications $(>40 \%)$ is shown above each node. These proteins were divided into seven Clades, and were represented by different colors, respectively. 
A Phylogenetic Tree

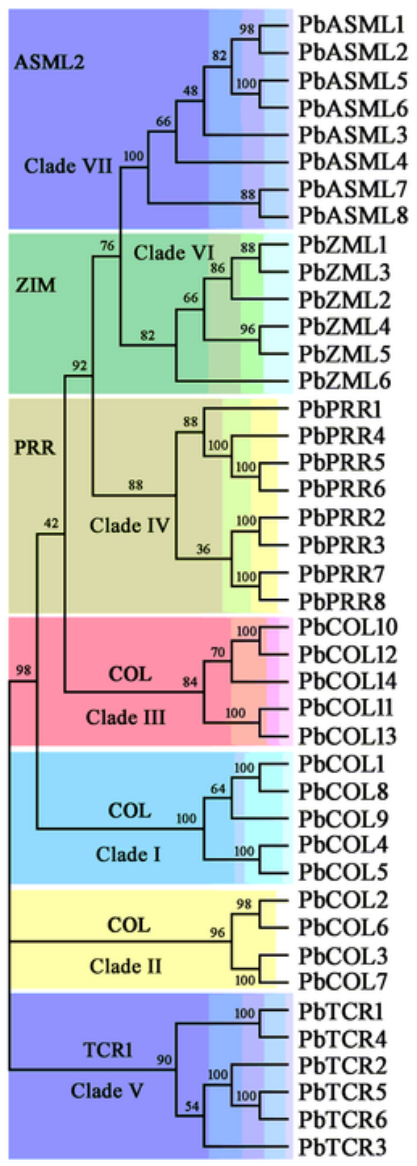

B Expression Profiles

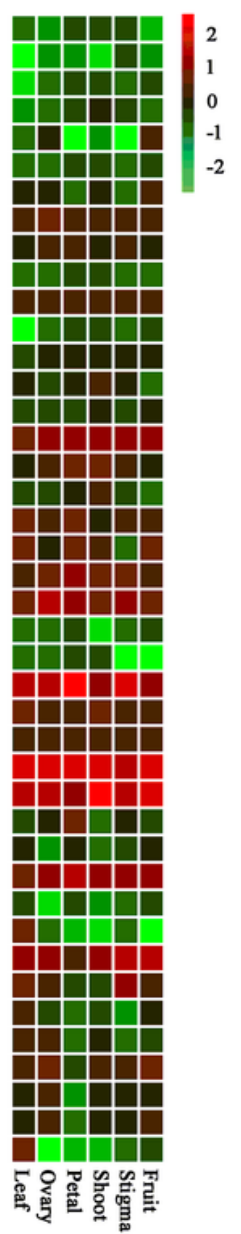

C Gene Structure
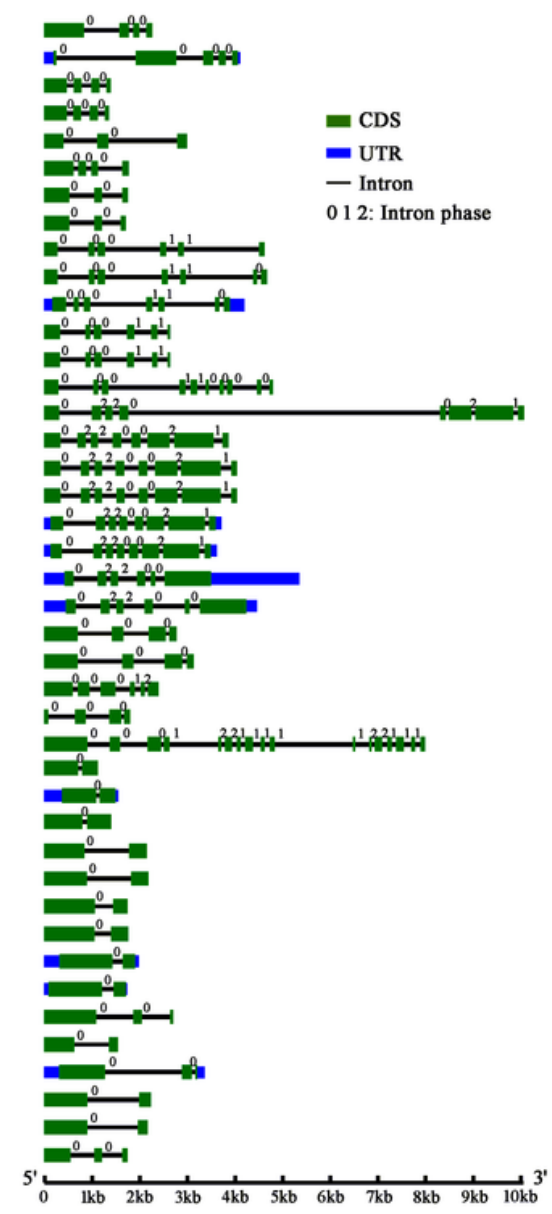

D Protein Structure

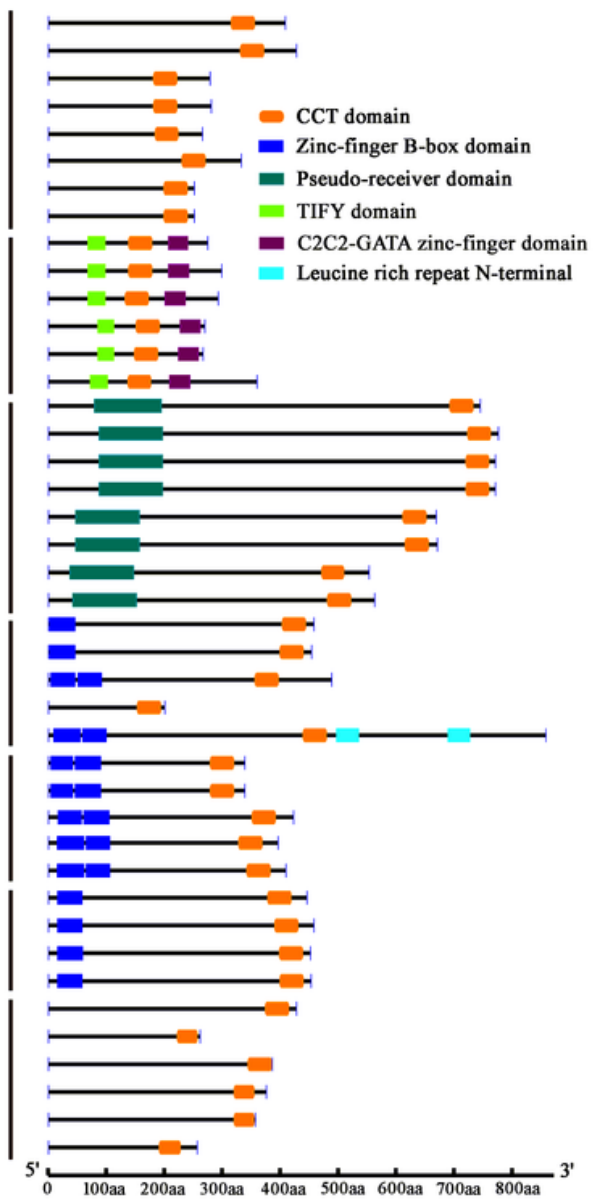

Figure 4

Phylogenetic relationships, expression profiles, gene structure and protein structure of pear CCT genes. (A) Neighbor-joining trees constructed for CCT genes from the ASML2, ZIM, PRR, COL and TCR1 families. (B) Heatmap showing the expression profiles of CCT genes in different tissues, including leaf, ovary, petal, shoot, stigma and fruit. Color gradient from red-to-green indicates expression values change from high to low. (C) Structure of CCT genes with exon(s) in green, UTR regions in blue, and solid lines between the colored boxes indicating introns. The number indicates the phases of the corresponding introns. (D) Structures of CCT proteins with the CCT DNA binding domains represented by orange boxes, the Zincfinger B-box domain in royalblue, Pseudo-receiver domain in aerugo, TIFY domain in green, C2C2-GATA zinc-finger domain in purple and Leucine rich repeat $\mathrm{N}$-terminal domain represented by sky-blue boxes. 


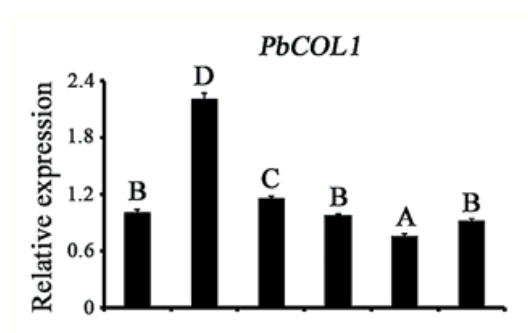

PbCOL 7

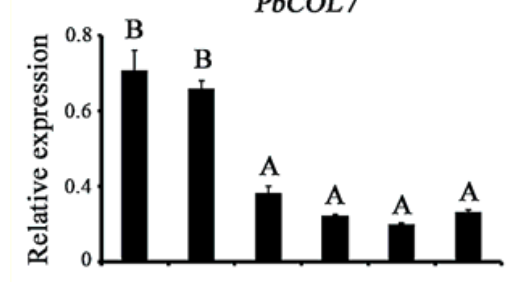

PDPRR 3

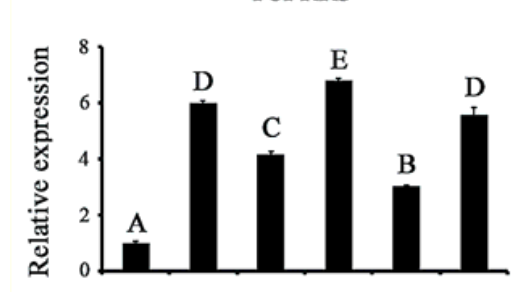

PbTCR2

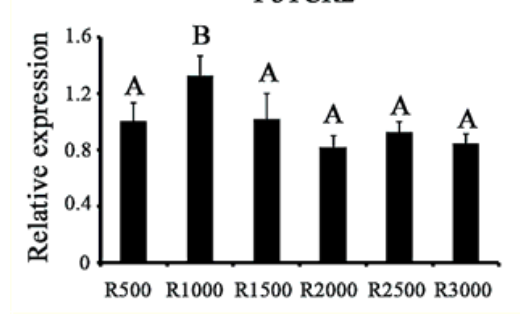

PbCOL 3

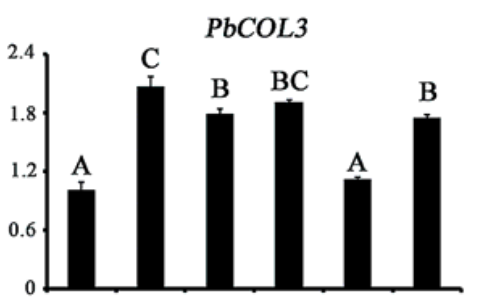

PbCOL 8

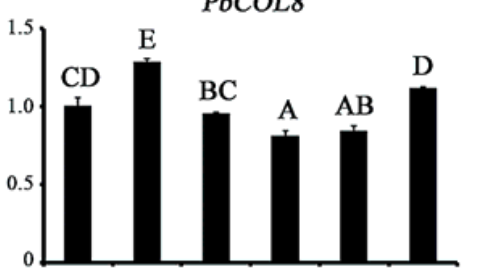

PbPRR4

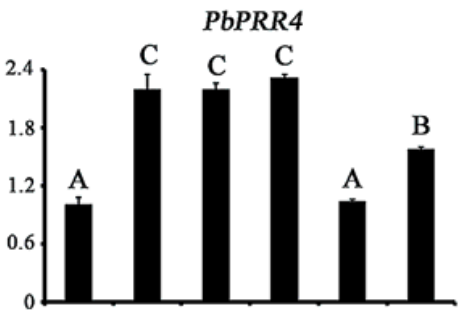

PbTCR3

PbCOL5

PbCOL6
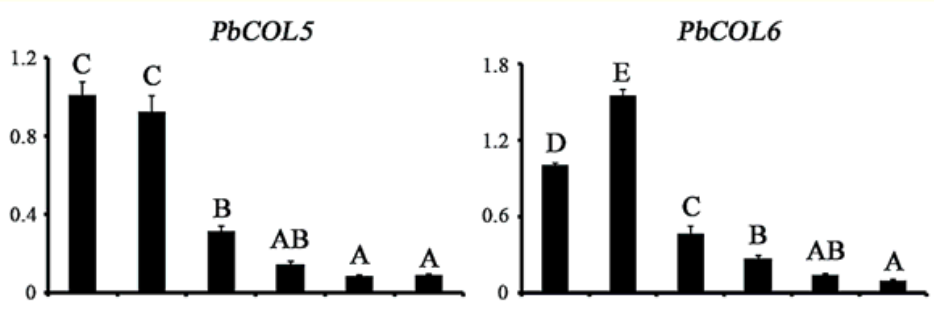

PbCOL11

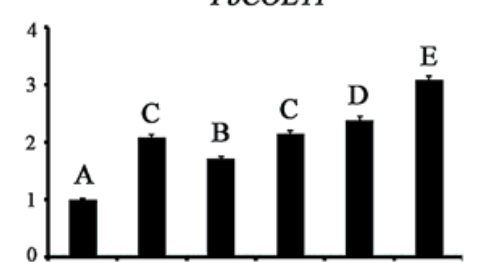

PbPRR8

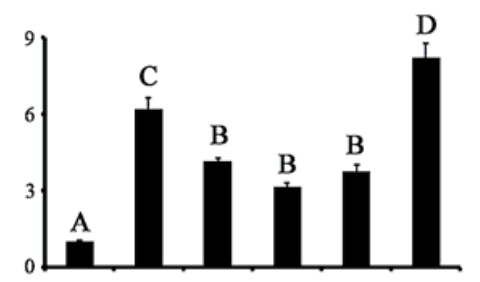

PbPRR2

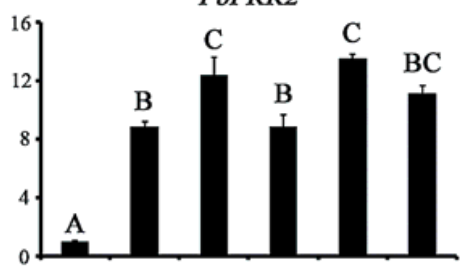

PbTCR1
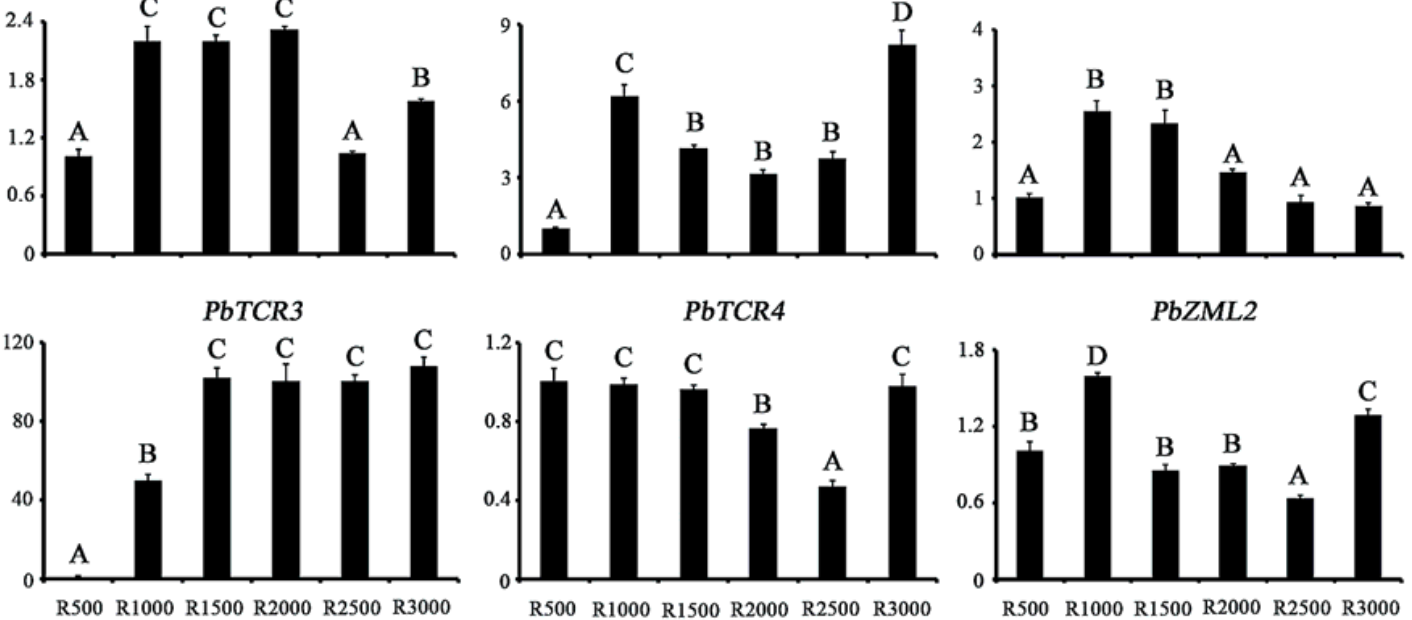

PbTCR4

PbZML2

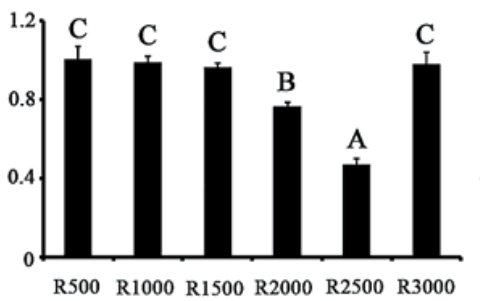

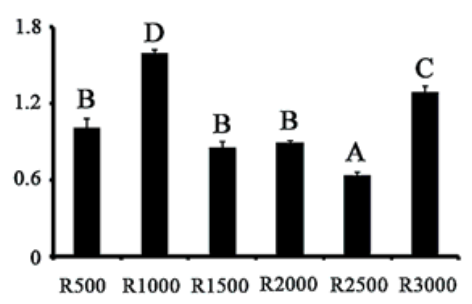

Figure 5

Expression profiles of 16 selected $\mathrm{PbCCT}$ genes under varying red light signals environments. Different capital letters above the bars indicate significant difference at $\mathrm{P}<0.01$.

R500/1000/1500/2000/2500/3000 (x-axis) indicate six red light intensity gradients, i.e., 500 Ix, 1000 Ix, $1500 \mathrm{Ix}, 2000 \mathrm{Ix}, 2500 \mathrm{~lx}$ and $3000 \mathrm{Ix}$. 
PbCOL1

PbCOL3

PbCOL5

PbCOL6

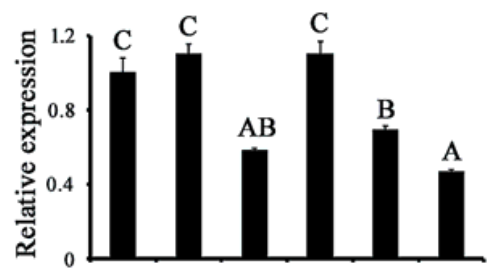

PbCOL7

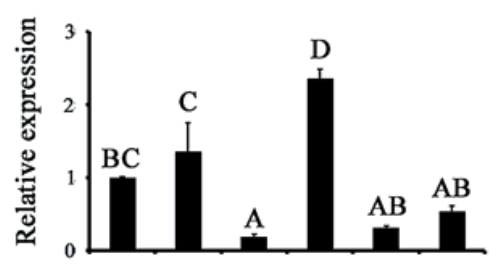

PbPRR3

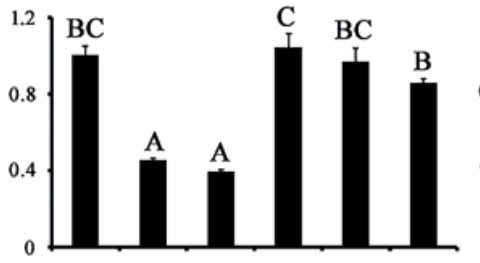

PbCOL8

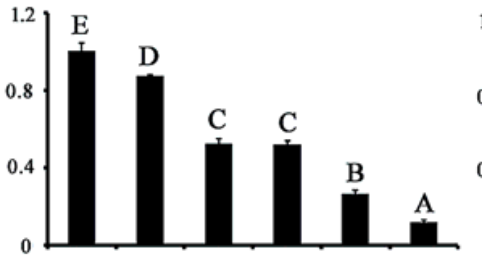

PbCOL11

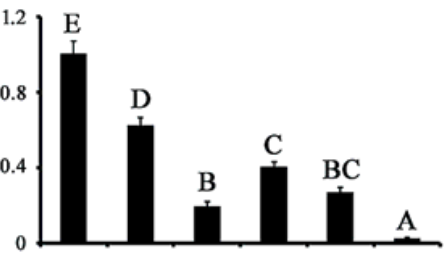

PbPRR2

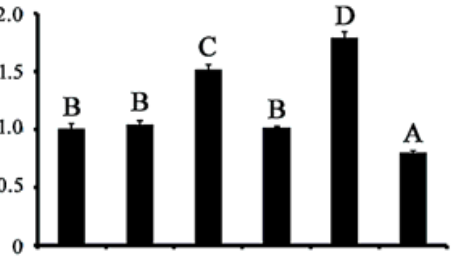

PbPRR4

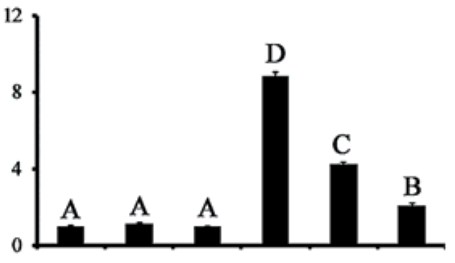

PbPRR8

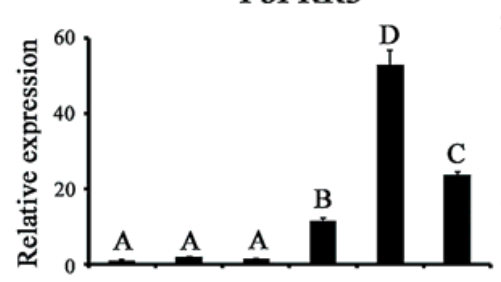

PbTCR2

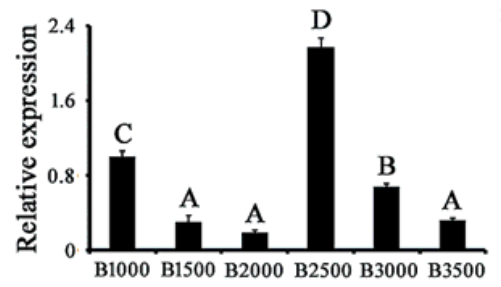

A

PbTCR 3
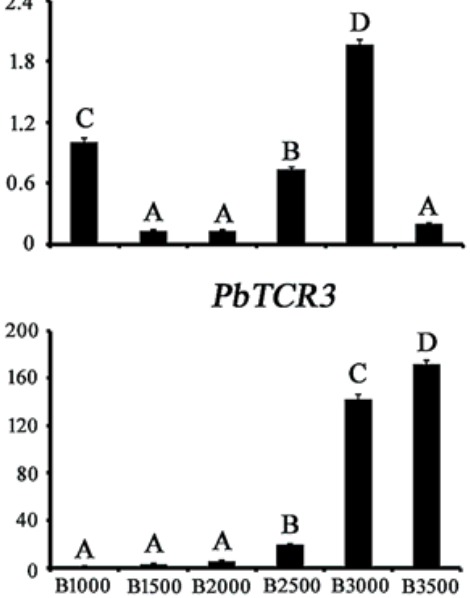

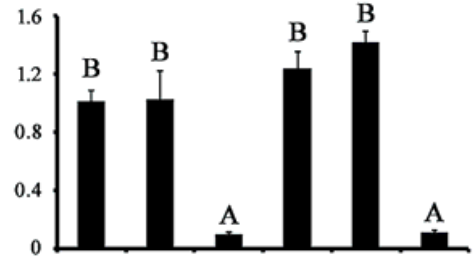

PbTCR4

.

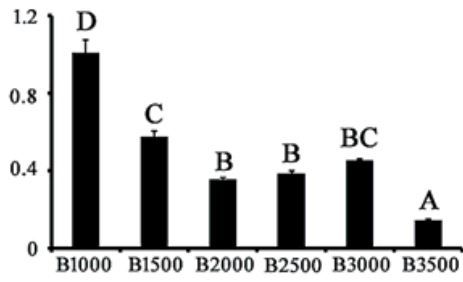

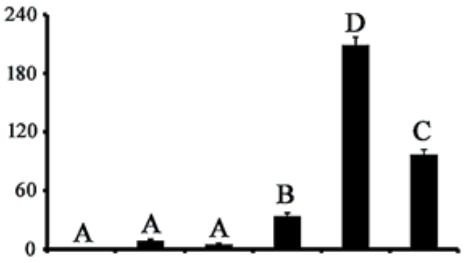

PbTCR1

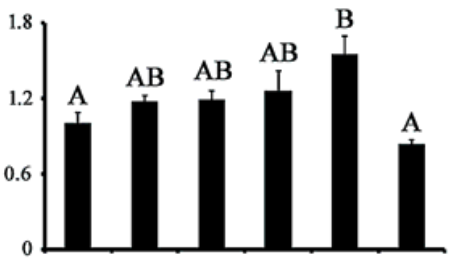

PbZML2

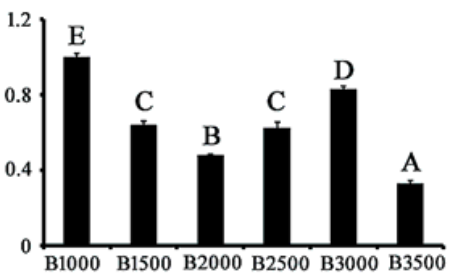

Figure 6

Expression profiles of 16 selected $\mathrm{PbCCT}$ genes under varying blue light signals environments. Different capital letters above the bars indicate significant difference at $P<0.01$.

B1000/1500/2000/2500/3000/3500 (x-axis) indicate six blue light intensity gradients, i.e., 1000 Ix, 1500 Ix, $2000 \mathrm{Ix}, 2500 \mathrm{~lx}, 3000 \mathrm{~lx}$ and $3500 \mathrm{~lx}$. 

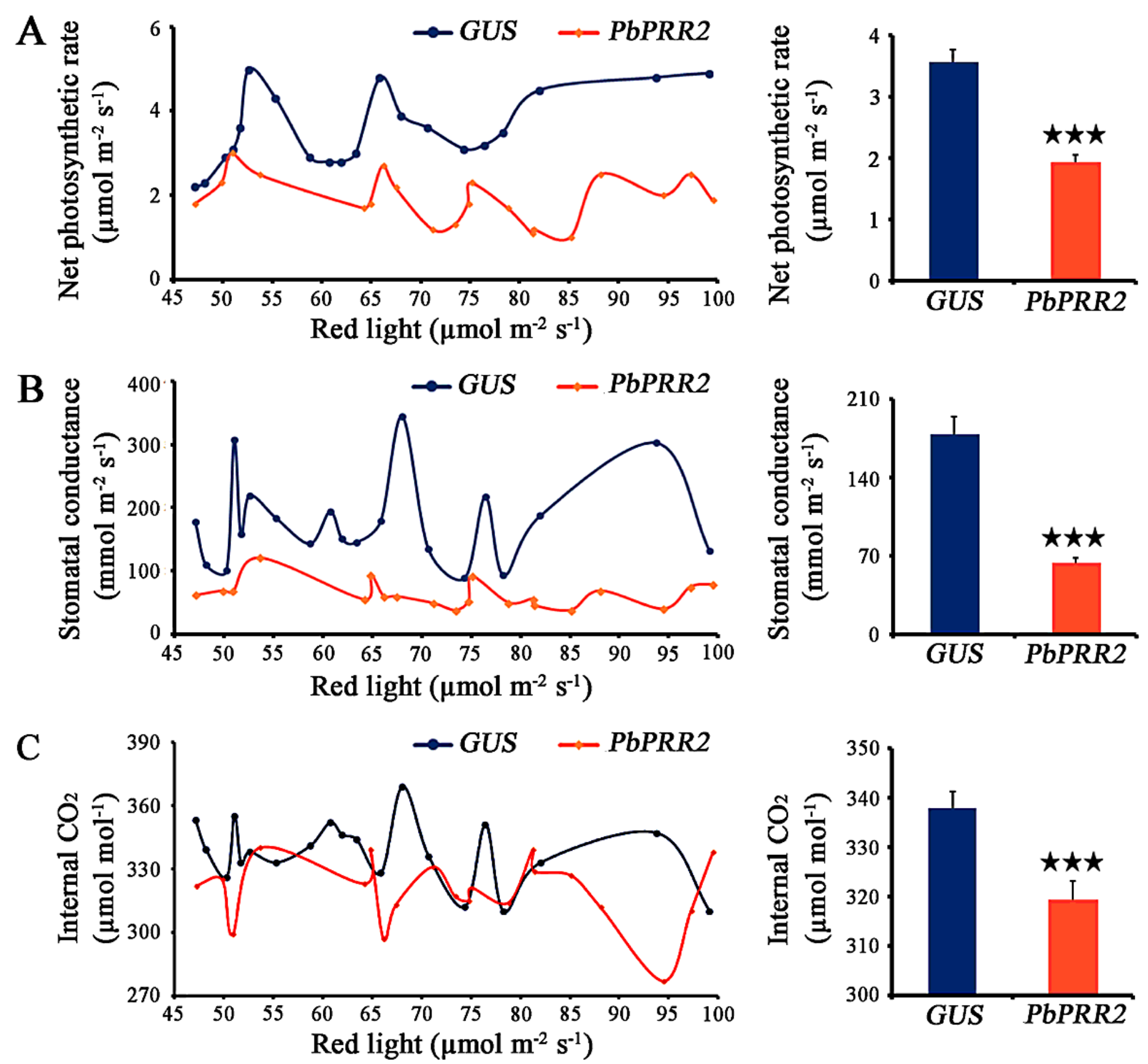

Figure 7

Photosynthetic performance of PbPRR2 transiently expressed in tobacco with the increasing R light intensity. Leaves were infiltrated with PbPRR2 (red line) or the control pHEX2-GUS (blue line). Data for three key photosynthetic parameters, i.e., net photosynthetic rate (A), stomatal conductance (B) and internal CO2 $(C)$ are presented as mean \pm SEM $(n=20)$. Asterisks indicate significant difference at $P<0.001$.

\section{Supplementary Files}

This is a list of supplementary files associated with this preprint. Click to download. 
- Additionalfile1.xIsx

- Additionalfile2.xIsx

- Additionalfile3.xIsx

- Additionalfile4.xIsx

- Additionalfile5.tif

- Additionalfile6.tif 